countries are experiencing an increasing prevalence of chronic diseases due to ageing population and lifestyle factors. Our paper aims to review the association between chronic diseases and risk of workplace injuries, and propose a health-based risk assessment framework which would incorporate employees' health factors into the risk assessment process which employers are familiar with.

Methods We first reviewed the evidence for the association between chronic diseases and risk of injuries in the workplace through literature review. Subsequently, we proposed a healthbased risk assessment framework that incorporates employees' health factors, giving examples on how this can be done, and at the same time highlight some of the challenges.

Results Many recent studies found that increased body mass index was associated with higher risk of injuries, while older workers were at risk of more severe injuries. Diabetes mellitus was also associated with workplace injuries in some studies. There were fewer studies looking at other chronic diseases such as hypertension. Our proposed health-based risk assessment framework incorporates employees' health into the risk assessment process outlined in the current Code of Practice for Risk Assessment, in a similar way as that for occupational health and safety hazards.

Discussion A health-based risk assessment framework can improve the integration of health and safety and minimise the need to have different methods of managing health and safety.

\section{ANALYSIS OF UNSTABLE CHROMOSOMAL ABERRATIONS FREQUENCY, MICRONUCLEI TEST, HAEMATOLOGICAL PARAMETERS AND RECEIVED DOSES BY PROFESSIONALS EXPOSED TO IONISING RADIATION}

\begin{abstract}
1,2Jelena Djokovic*, ${ }^{3}$ Jadranko Simic, ${ }^{4}$ Vojislav Antic, ${ }^{1,5}$ Milan Gajic, ${ }^{1,2}$ Aleksandar Milovanovic, ${ }^{1,2}$ Petar Bulat. 'School of Medicine, University of Belgrade, Belgrade, Serbia; 'Serbian Institute of Occupational Health 'Dr Dragomir Karajovic', Belgrade, Serbia; ${ }^{3}$ South East Europe Consultants - SEEC, Belgrade, Serbia; ${ }^{4}$ Centre for Nuclear Medicine, Clinical Centre of Serbia, Belgrade, Serbia; ${ }^{5}$ Institute of Medical Statistics and Informatics, Belgrade, Serbia
\end{abstract}

\subsection{6/oemed-2018-ICOHabstracts. 1081}

Introduction Ionising radiation is often used in medicine for diagnostic and therapeutic purposes. Radiation workers exposed to ionising radiation have to follow all the safety measures and precautions at their work. The purpose of this article was to analyse the impact of low-dose ionising radiation to medicine professionals exposed to ionising radiation of the Clinical Centre of Serbia.

Methods Data from the last medical check-ups, obtained from the medical records of 148 employees from Clinical centre of Serbia, were analysed. They were divided into tree groupsempoyees in nuclear medicine, in interventional radiology and general radiology. A typical check-up, haematological parameters analysis, as well as special cytogenetical analyses, such as unstable chromosomal aberrations and micronucleus test, were carried out. The received cumulative 5 year dose was measured by personal inactive thermoluminescent dosimeters calibrated into personal doses equivalent Hp (10).

Results By comparing the results of the frequency of unstable chromosomal aberrations in the last periodical check-up of the employees in all the three groups we have found that the nuclear medicine employees had a significantly higher frequency of unstable chromosomal aberrations $\left(x^{2}=6.634\right.$; $\mathrm{p}<0.05)$. Employees in nuclear medicine had significantly lower levels of red blood cells as compared to employees in general and interventional radiology. Statistically higher platelet count was ascertained with the employees in general radiology (ANOVA, p <0.01). Nuclear medicine employees have received statistically higher cumulative dose than the employees in interventional radiology and general radiology $\left(x^{2}=23.465\right.$; $\mathrm{p}<0.01)$. There is also no statistically significant difference in the outcome of the micronucleus test between groups of employees $\left(x^{2}=1.245 ; \mathrm{p}>0.05\right)$.

Discussion Out of the three groups of employees tested, nuclear medicine employees have increased health risks than employees in interventional radiology and general radiology. There is a need to monitor their health condition by periodical check-ups for prevention from occupational diseases.

\section{THUNDERSTORM ASTHMA IN THE MEDITERRANEAN AREA: SEASONAL WEATHER AND EFFECT ON HEALTH FOR OUTDOOR WORKERS}

${ }^{1} \mathrm{G}$ D'Amato, ${ }^{2}$ Annesi-Maesano, ${ }^{3} \mathrm{C}$ Grandi, ${ }^{4,5} \mathrm{~L}$ Cecchi, ${ }^{3} \mathrm{MC} \mathrm{D}^{\prime}$ Ovidio*.${ }^{1}$ Division of Respiratory and Allergic Diseases, Department of Chest Diseases, High Speciality A. Cardarelli Hospital, Naples, Italy; ${ }^{2}$ Epidemiology of Allergic and Respiratory Diseases Department (EPAR), Pierre Louis Institute of Epidemiology and Public Health (IPLESP UMRS1136), UPMC Univ Paris 06, INSERM, Saint-Antoine Medical School, Sorbonne Universités, Paris, France; ${ }^{3}$ National Workers' Compensation Authority (INAIL), Department of Occupational and Environmental Medicine, Epidemiology and Hygiene, Monte Porzio Catone (RM), Italy; ${ }^{4}$ UOSD Allergy and Clinical Immunology, USL4 Prato, Italy; 5 Interdepartmental Centre of Bioclimatology, University of Florence, Florence, Italy

\subsection{6/oemed-2018-ICOHabstracts. 1082}

Introduction An increasing body of evidence shows the occurrence of asthma epidemics, sometimes also severe, during thunderstorms in the pollen season, in various geographical zones. Thunderstorms have been linked to asthma epidemics and there are descriptions of asthma outbreaks associated with thunderstorms in several cities, prevalently Australia (Melbourne) and Europe (in UK Birmingham and London, and in Italy in Naples). Considering this background it is important to promote the dissemination of these evidence also in occupational setting.

Methods Analysis of literature was performed on asthma, thunderstorm, occupational health. Description of these events in the Mediterranean area was also investigated.

Results Asthma outbreaks associated with thunderstorms occurred in several cities as London (24/25 June 1994) and Melbourne (21 November 2016). In the first outbreak 640 patients with asthma or other airway diseases attended London hospitals (nearly 10 times the usual number); in Melbourne, hospitals were swamped with emergency patients affected by severe asthma attacks (more than 8500 patients across Monday night and Tuesday and 8 died). The frequency of thunderstorms had recently increased in some geographical areas, particularly in temperate and subtropical climates. Research has shown that thunderstorms are expected to become more severe as the climate changes.

Conclusion Thunderstorms have been linked to asthma epidemics, especially during the pollen seasons. There is evidence that hat under wet conditions or during thunderstorms, pollen grains, release into the atmosphere their content that can reach lower airways inducing asthma reactions.

All individuals affected by pollen allergy should be alerted to the danger of being outdoors during a thunderstorm in the 
pollen season. Outdoor workers should be informed; training should be addressed in order to promote collective and individual control and preventive measures. Being the Mediterranean area subject to thunderstorm episodes it is necessary the activation to specific planning to respond at these events.

\section{NETWORKS FOR POLLEN AND FUNGAL SPORES MONITORING: INTEGRATED APPROACH TO SUPPORT HEALTH SURVEILLANCE WITH REGARD TO OCCUPATIONAL ALLERGIES IN ITALY}

\begin{abstract}
${ }^{1} \mathrm{MC}$ D'Ovidio*, ${ }^{2} \mathrm{~F}$ de' Donato, ${ }^{3} \mathrm{R}$ Ariano, ${ }^{4,5} \mathrm{M}$ Bonini, ${ }^{3} \mathrm{G}$ Moscato, ${ }^{6} \mathrm{~V}$ De Gironimo, ${ }^{1} \mathrm{~S} \mathrm{Di}$ Renzi, ${ }^{7} \mathrm{M}$ Bonafede, ${ }^{1} \mathrm{C}$ Grandi, ${ }^{7} \mathrm{~A}$ Marinaccio, ${ }^{2} \mathrm{P}$ Michelozzi. 'National Workers' Compensation Authority (INAIL), Department of Occupational and Environmental Medicine, Epidemiology and Hygiene, Monte Porzio Catone (Rome), Italy; ${ }^{2}$ Department of Epidemiology Lazio Regional Health Service, ASL Roma 1, Rome, Italy; ${ }^{3}$ Association of Italian Immunologists, Outpatient and Hospital Allergists (A.A.I.I.T.O.), Florence, Italy; ${ }^{4}$ Department of Medical Prevention, Agenzia di Tutela della Salute della Città Metropolitana di Milano, Parabiago (Milan) Italy; ${ }^{5}$ Italian Monitoring Network in Aerobiology of the Italian Association of Aerobiology (A.I.A.- R.IIM.A. ${ }^{\circledR}$ ), Parabiago (Milan), Italy; ${ }^{6}$ POLLnet Network, Italian National Institute for Environmental Protection and Research (ISPRA), Rome, Italy; ${ }^{7}$ National Workers' Compensation Authority (INAIL), Department of Occupational and Environmental Medicine, Epidemiology and Hygiene, Rome, Italy
\end{abstract}

\subsection{6/oemed-2018-ICOHabstracts.1083}

Introduction The purpose of national and international aerobiological networks is the monitoring of pollen and fungal spores in defined geographical areas, followed by the spreading of data regarding several seasonal species by the use of the bulletins and calendars. These data are usually available on line. Our proposal focuses on the integration of exposure data with health surveillance systems (ER visits and hospital admissions) and health surveillance practices in workers occupationally exposed to aeroallergens.

Methods Within an Italian project funded by the Ministry of Health, the monitoring of environmental exposures, including pollen in relation to health outcomes is being set up to evaluate the effects and set up surveillance and prevention measures. For the major Italian cities, pollen data from the different monitoring networks and health outcome data to set up a rapid surveillance system with weekly updates on the potential health effects of pollen and fungal spores among vulnerable groups such as people suffering from allergies and respiratory conditions, children, workers etc.

Results The results will help provide a rapid monitoring on the health effects of pollen exposure in 2017 in Italy. Specific focus will be on occupational exposure and subjects with asthma and allergic rhinitis. These findings will also serve as a basis for the setting up of specific epidemiological studies to increase the evidence on health risks and develop adequate prevention measures.

Conclusion The optimisation of environmental and health data acquisition is of crucial importance for a proper analysis of trends regarding health outcomes in a number of subjects employed in different job sectors and with different job titles. Networks of data provided by several institutions may facilitate a better understanding of the results of epidemiological studies on occupational allergies, allowing a better study design in the case of both general populations and workers exposed to aeroallergens.
1177 HOME-OFFICE: NEW CHALLENGES FOR OCCUPATIONAL HEALTH AND SAFETY

${ }^{1}$ C Esteban*, ${ }^{1}$ CA Fiori, ${ }^{2}$ PA Ramadan, ${ }^{1}$ EC Sá, ${ }^{1} \mathrm{MJ}$ Gimenez. ${ }^{1} \mathrm{ABC}$ Medical School Faculty, Santo André, Brazil; ${ }^{2}$ University of São Paulo, São Paulo, Brazil

\subsection{6/oemed-2018-ICOHabstracts. 1084}

Introduction The domestic office is a new and constantly growing labour organisation in Brazil and the world, especially in the tertiary or service sector. In 2011, the Brazilian Law began to recognise the existence of telework, which represented an important step in regulating this new type of work. However, it is still very difficult to define what characterises remote work and to guarantee the labour rights provided for in the law on health and safety at work. The objective of this study was to review the contents of health and safety in the field of teleworking and the current regulatory norms.

Methods Bibliografic review at Pubmed, LILACS and MEDLINE databases, using the key-words 'home-office', 'workorganization' and 'labour law', in the interval of 1980 to 2016.

Results Home office work has many good points: inclusion of people with disabilities, reduction of operational costs and bureaucratic expenses by the employer, self-determination of working time and the reduction of travel time by the employee. Nowadays, the labour rights of those who perform their work at a distance are the same as those who go to the company headquarters, which will be responsible for occupational diseases and even accidents.

Conclusion The courts maintained a conservative view of homeoffice work in Brazil. In theory, this type of work must follow the labour law of formal work, being responsible for occupational diseases and even accidents. However, the big question is how to put all these guidelines into practice, how to supervise the duties of employers and employees, and how to guarantee labour rights, such as work accidents. Therefore, it is necessary to deepen our studies on the subject and make it legally established.

\section{HEALTH EFFECTS FROM HAND-ARM VIBRATIONS IN SWEDISH MECHANICAL INDUSTRY}

Per Vihlborg, Ing-Liss Bryngelsson, Louise Fornander, Pål Graff. Department of Occupational and Environmental Medicine, Örebro University Hospital

\subsection{6/oemed-2018-ICOHabstracts. 1085}

Background Working with handheld vibrating tools is common in the mechanical industry and one of the main causes of occupational disease in Sweden. There are several well-known effects on vascular, neurological and musculoskeletal symptoms in the hands from vibrations.

Methods This report is based on medical examinations performed on 38 males at a mechanical industry. The medical examination consisted of questionnaires regarding symptoms and exposure, standardised medical examination and quantitative sensory testing (QST). The exposure to hand-arm vibrations was also measured. Blood samples were collected to investigate for biological markers for vibration exposure.

Results $24 \%$ of the exposed subjects had vascular symptoms in form of Raynaud's phenomenon, and 37\% had increased cold intolerance. More common was neurological symptoms which affected nearly $60 \%$ of the exposed subject. Although the 